\title{
Dystonic drop foot gait in a patient with manganism
}

回舟

In 1983-1984, a 29-year-old battery factory worker gradually developed gait difficulties and tremor. In 1984, he was found to have severely elevated serum manganese levels and retired from work. Clinical examination in 1984 (videos 1-3 on the Neurology ${ }^{\circledR}$ Web site at www.neurology.org) showed bilateral foot dystonia and profound parkinsonism. His gait was shuffling, although he lifted his knees relatively highly. Strength of foot dorsiflexors was normal. He did not respond to dopaminergic drugs and his gait disorder slowly deteriorated to an asymmetric dystonic drop foot gait (videos 4 and 5, recorded in 2008).

Although the walking pattern of this patient with manganism resembled that of a strutting rooster, it was distinct from cock gait as classically described in manganism. Patients with classic cock gait walk on the metatarsophalangeal joints and their heels do not touch the ground. ${ }^{1,2}$

Jules Janssens, MD, Wim Vandenberghe, MD, PhD, Leuven, Belgium

Address correspondence and reprint requests to Dr. Wim Vandenberghe, Department of Neurology, University Hospitals Leuven, Herestraat 49, 3000 Leuven, Belgium; wim.vandenberghe@uzleuven.be

Disclosure: The authors report no disclosures.

\section{Supplemental data at} www.neurology.org

\section{ACKNOWLEDGMENT}

W.V. is a Senior Clinical Investigator of the Fund for Scientific Research Flanders (FWO).

1. von Jaksch R. Ueber Mangantoxikosen und Manganophobie. Münch Med Wochenschr 1907;20:969-972.

2. Huang CC, Chu NS, Lu CS, Calne DB. Cock gait in manganese intoxication. Mov Disord 1997;12:807-808. 


\title{
Neurology
}

\author{
Dystonic drop foot gait in a patient with manganism \\ Jules Janssens and Wim Vandenberghe \\ Neurology 2010;75;835 \\ DOI 10.1212/WNL.0b013e3181f0752d
}

This information is current as of August 30, 2010

$\begin{array}{ll}\begin{array}{l}\text { Updated Information \& } \\ \text { Services }\end{array} & \begin{array}{l}\text { including high resolution figures, can be found at: } \\ \text { http://n.neurology.org/content/75/9/835.full }\end{array} \\ \text { Supplementary Material } & \text { Supplementary material can be found at: } \\ \text { http://n.neurology.org/content/suppl/2010/08/27/75.9.835.DC1 } & \\ \text { This article cites } 2 \text { articles, } 0 \text { of which you can access for free at: } \\ \text { http://n.neurology.org/content/75/9/835.full\#ref-list-1 } \\ \text { This article, along with others on similar topics, appears in the } \\ \text { following collection(s): } \\ \text { Basal ganglia } \\ \text { http://n.neurology.org/cgi/collection/basal_ganglia } \\ \text { Dystonia } \\ \text { http://n.neurology.org/cgi/collection/dystonia } \\ \text { Gait disorders/ataxia } \\ \text { http://n.neurology.org/cgi/collection/gait_disorders_ataxia } \\ \text { Other toxicology } \\ \text { http://n.neurology.org/cgi/collection/other_toxicology } \\ \text { Parkinson's disease/Parkinsonism } \\ \text { http://n.neurology.org/cgi/collection/parkinsons_disease_parkinsonism } \\ \text { Information about reproducing this article in parts (figures,tables) or in } \\ \text { its entirety can be found online at: } \\ \text { http://www.neurology.org/about/about_the_journal\#permissions } \\ \text { Information about ordering reprints can be found online: } \\ \text { http://n.neurology.org/subscribers/advertise }\end{array}$

Neurology ${ }^{\circledR}$ is the official journal of the American Academy of Neurology. Published continuously since 1951, it is now a weekly with 48 issues per year. Copyright . All rights reserved. Print ISSN: 0028-3878. Online ISSN: 1526-632X.

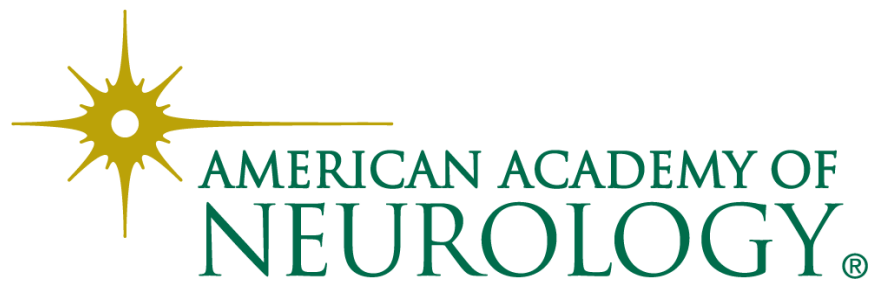

